\title{
DISORDERED BOSE EINSTEIN CONDENSATES WITH INTERACTION
}

\author{
ROBERT SEIRINGER, JAKOB YNGVASON, AND VALENTIN A. ZAGREBNOV
}

\begin{abstract}
We study the effects of random scatterers on the ground state of the one-dimensional Lieb-Liniger model of interacting bosons on the unit interval in the Gross-Pitaevskii regime. We prove that Bose Einstein condensation survives even a strong random potential with a high density of scatterers. The character of the wave function of the condensate, however, depends in an essential way on the interplay between randomness and the strength of the two-body interaction. For low density of scatterers or strong interactions the wave function extends over the whole interval. High density of scatterers and weak interaction, on the other hand, leads to localization of the wave function in a fragmented subset of the interval.
\end{abstract}

\section{INTRODUCTION}

While the effects of random potentials on single particle Schrödinger operators [1] and ideal Bose gases [2, 3, 4, are rather well explored, the present understanding of such effects on many-body systems of interacting particles is much less complete. In recent years, however, many papers concerning the interplay of Bose-Einstein Condensation (BEC) and disorder have appeared of which references [5]-18] are but a sample.

We present here results on a model that is in a sense the simplest one imaginable where this interplay can be studied by rigorous mathematical means. This is the one-dimensional Lieb-Liniger (LL) model 20] of bosons with contact interaction in a 'flat' trap, augmented by an external random potential that is generated by Poisson distributed point scatterers of equal strength. We study the ground state and prove that, no matter the strength of the random potential, BEC is not destroyed by the random potential in the Gross-Pitaevskii (GP) limit where the particle number tends to infinity while the coupling parameter in a mean-field scaling stays fixed. The character of the wave function of the condensate, however, depends in an essential way on the relative size of the three parameters involved. These are the scaled coupling parameter $\gamma$ for the interaction among the particles, the density of the scatterers $\nu$, and the strength $\sigma$ of the scattering potential. All these parameters are assumed to be large in suitable units.

Our main results an be summarized as follows: For $\sigma \gg 1$ we identify three different "phases" of the condensate. For large $\gamma \gg \nu^{2}$ the condensate is extended over the whole trap (the unit interval in our model). A transition from a delocalized

\footnotetext{
Key words and phrases. Bose Einstein condensation; random Hamiltonians; one-dimensional models.

Contribution to the proceedings of ICMP12, Aalborg, Denmark, August 6-11, 2012. (C) 2012 by the authors. This paper may be reproduced, in its entirety, for non-commercial purposes.
} 
to a localized state takes place when $\gamma$ is of the order $\nu^{2}$, in the sense that for $\gamma \ll \nu^{2}$ the density is essentially distributed among a fraction $\lambda \ll 1$ of the $\nu \gg 1$ intervals between the obstacles. For $\gamma \gg \nu /(\ln \nu)^{2}$ we still have $\lambda \nu \gg 1$, but for $\gamma \sim \nu /(\ln \nu)^{2}$ the fraction of intervals that are significant occupied shrinks to $O\left(\nu^{-1}\right) 1$ We stress, however, that in all cases there is complete BEC into a single state in the limit when the particle number tends to infinity.

Details of our analysis are given in the paper [19].

\section{The Model}

The model we consider is the Lieb-Liniger model of bosons with contact interaction on the unit interval but with an additional external random potential $V_{\omega}$. The Hamiltonian on the Hilbert space $L^{2}([0,1], d z)^{\otimes_{\mathrm{s}} N}$ is

$$
H=\sum_{i=1}^{N}\left(-\partial_{z_{i}}^{2}+V_{\omega}\left(z_{i}\right)\right)+\frac{\gamma}{N} \sum_{i<j} \delta\left(z_{i}-z_{j}\right)
$$

with $\gamma \geq 0$ and Dirichlet boundary conditions at the end points of the interval. The random potential is taken to be

$$
V_{\omega}(z)=\sigma \sum_{j} \delta\left(z-z_{j}^{\omega}\right)
$$

with $\sigma \geq 0$ independent of the random sample $\omega$ while the obstacles $\left\{z_{j}^{\omega}\right\}$ are Poisson distributed with density $\nu \gg 1$, i.e., their mean distance is $\nu^{-1}$.

The Hamiltonian (11) can be defined rigorously via the quadratic form on the Sobolev space $H^{1}\left([0,1]^{\times N}\right)$ given by the expression on the right hand side of (10), noting that functions in the Sobolev space can be restricted to hyperplanes of codimension 1. See also 21, Ch. 14.6. The limiting case $\sigma=\infty$ amounts to requiring the wave function to vanish at the positions of the obstacles $z_{j}^{\omega}$.

There are several reasons for studying this model:

- It is the simplest model of its kind.

- BEC in the ground state can be proved in a suitable limit, for an arbitrary nonnegative external potential.

- $V_{\omega}$ is simple enough to allow a rigorous analysis of the condensate.

We remark also that the case $\gamma=0, \sigma=\infty$ corresponds to the Luttinger-Sy model 22 .

Once BEC has been established the main question concerns the dependence of the properties of the condensate on the three parameters $\gamma, \sigma$ and $\nu$.

\section{BEC in the Ground State}

A basic nontrivial fact about the model (1), whose proof will be discussed below, is that for fixed $\gamma, \sigma$ and configuration $\omega$ there is complete BEC in the ground state in the sense that the 1-particle density matrix/ $N$ converges to a one dimensional projector as $N \rightarrow \infty$. As usual, the 1-particle density matrix of the ground state wave function $\Psi_{0}$ is defined as

$$
\gamma_{0}\left(z, z^{\prime}\right)=N \int \Psi_{0}\left(z, z_{2}, \ldots, z_{N}\right) \overline{\Psi_{0}\left(z, z_{2}, \ldots, z_{N}\right)} d z_{2} \cdots d z_{N} .
$$

\footnotetext{
${ }^{1}$ The notation $a \sim b$ means that $a / b$ tends to a finite, nonzero constant.
} 
Furthermore, the corresponding wave function of the condensate, i.e., the eigenfunction to the highest eigenvalue $O(N)$ of the integral operator defined by $\gamma\left(z, z^{\prime}\right)$ is, in the limit $N \rightarrow \infty$, the $L^{2}$-normalized minimizer of the Gross-Pitaevskii (GP) energy functional

$$
\mathcal{E}^{\mathrm{GP}}[\psi]=\int_{0}^{1}\left\{\left|\psi^{\prime}(z)\right|^{2}+V_{\omega}(z)|\psi(z)|^{2}+(\gamma / 2)|\psi(z)|^{4}\right\} d z .
$$

Formally, (41) is obtained by computing the expectation value of $H / N$ with a Hartree-type wave function $\Psi=\psi^{\otimes N}$ and taking the limit $N \rightarrow \infty$.

Since we want to consider large values of $\nu, \sigma$ and $\gamma$ it is important to estimate also the rate of the convergence of the 1-particle density matrix as $N \rightarrow \infty$, in dependence of these parameters and of the configurations $\omega$.

Our proof of BEC in the GP limit is simpler than the corresponding proof in three or two dimensions 23 because the one-dimensional case considered here corresponds to a high density, mean-field limit. In contrast, the work in 23] deals with a low density limit that requires quite different tools.

3.1. The Proof of BEC (sketch). The proof of BEC is based on energy bounds:

- An upper bound to the many-body ground state energy $E_{0}^{\mathrm{QM}}$ by taking $\psi_{0}^{\otimes N}$ as a trial function for $H$ where $\psi_{0}$ is the minimizer of the GP energy functional, normalized so that $\left\|\psi_{0}\right\|_{2}=1$. This gives

$$
E_{0}^{\mathrm{QM}} \leq N e_{0}
$$

where $e_{0}=\mathcal{E}^{\mathrm{GP}}\left[\psi_{0}\right]$ is the g.s.e. of the GP functional.

- An operator lower bound for the many-body Hamiltonian $H$, up to controlled errors, in terms of the 1-particle mean-field Hamiltonian

$$
h=-\partial_{z}^{2}+V_{\omega}(z)+\gamma\left|\psi_{0}(z)\right|^{2}-(\gamma / 2) \int\left|\psi_{0}\right|^{4}
$$

which has $\psi_{0}$ as ground state with energy $e_{0}$. For this bound similar ideas as in the proof of Proposition 6.4 in [24] are used.

BEC follows from the upper and lower bounds and the fact that there is an energy gap between $e_{0}$ and the next lowest eigenvalue, $e_{1}$, of the mean-field Hamiltonian $h$ :

Let

$$
N_{0}=\int \overline{\psi_{0}(z)} \gamma_{0}\left(z, z^{\prime}\right) \psi_{0}\left(z^{\prime}\right) d z d z^{\prime}=\left\langle\Psi_{0}\left|a^{\dagger}\left(\psi_{0}\right) a\left(\psi_{0}\right)\right| \Psi_{0}\right\rangle
$$

be the occupation number of the GP ground state $\psi_{0}$ in the many-body ground state $\Psi_{0}$. Then the energy bounds give

$$
N_{0} e_{0}+\left(N-N_{0}\right) e_{1}-o(1) N e_{0} \leq E_{0}^{\mathrm{QM}} \leq N e_{0}
$$

where the $o(1)$ factor depends only on $\gamma$ and $N$. This implies an upper bound for the depletion:

$$
\left(1-\frac{N_{0}}{N}\right) \leq o(1) \frac{e_{0}}{e_{1}-e_{0}}
$$

More precisely, the estimate on the depletion of the condensate proved in [19] is 
THEOREM 1 (BEC).

$$
\left(1-\frac{N_{0}}{N}\right) \leq C \frac{e_{0}}{e_{1}-e_{0}} N^{-1 / 3} \min \left\{\gamma^{1 / 2}, \gamma\right\} .
$$

We must, however, also consider the dependence of the energy gap, $e_{1}-e_{0}$, on the random potential and the parameters.

3.2. Remark. If $e_{0}<e_{1} \leq \cdots \leq e_{k}$ are the $(k+1)$ lowest eigenvalues of (6) with corresponding eigenfunctions $\psi_{j}$ and

$$
N_{<k}=\sum_{j=0}^{k-1}\left\langle\Psi_{0}\left|a^{\dagger}\left(\psi_{j}\right) a\left(\psi_{j}\right)\right| \Psi_{0}\right\rangle
$$

is the occupation of the $k$ lowest eigenstates with energies $\leq e_{k-1}$, then we have

$$
\left(1-\frac{N_{<k}}{N}\right) \leq C \frac{e_{0}}{e_{k}-e_{0}} N^{-1 / 3} \min \left\{\gamma^{1 / 2}, \gamma\right\}
$$

For finite $N$ this estimate may be more useful than (10) because even if $k \ll N$, $e_{k}-e_{0}$ can be substantially larger than $e_{1}-e_{0}$. Thus the right side of (12) can be $<1$ even in cases when (10) contains no information because the right-hand side is $>1$.

A situation when $1-N_{<k} / N$ is small for some $1 \ll k \ll N$ without $1-N_{0} / N$ being small is commonly referred to as a fragmented condensation. For finite $N$ this may be a reasonable substitute for the full condensation that in our model emerges in the large $N$ limit according to Theorem 3.1. We also point out that as far as the particle density $\rho(z)=\gamma(z, z)$ in position space is concerned, a fragmented condensate with non-overlapping single-particle wavefunctions is indistinguishable from a fully condensed state where the wavefunction of the condensate is a coherent superposition of the nonoverlapping functions. The difference shows up, however, in the density $\hat{\rho}(p)=(2 \pi)^{-1} \int \exp \left(\mathrm{i} p\left(z-z^{\prime}\right)\right) \gamma\left(z, z^{\prime}\right) d z d z^{\prime}$ in momentum space.

3.3. The energy gap. Consider a one-dimensional Schrödinger operator $-\partial_{z}^{2}+$ $W(z)$ on the unit interval with a nonnegative potential $W$ and Dirichlet boundary conditions.

LEMMA 1 (Gap). Define $\eta>0$ by $\eta^{2}=\pi^{2}+3 \int_{0}^{1} W(z) d z$. Then

$$
e_{1}-e_{0} \geq \eta \ln \left(1+\pi e^{-2 \eta}\right)
$$

The proof is based on a modification of a result of Kirsch and Simon 25, that involves the sup norm of $W$ instead of the integral. In our case $\eta=\eta_{\omega}=$ $\sqrt{\pi^{2}+3 m_{\omega} \sigma+3 \gamma}$ where $m_{\omega}$ is the number of obstacles in $[0,1]$, that is almost surely equal to $\nu$ in the limit $\nu \rightarrow \infty$.

For large $\sigma m_{\omega}$ the bound is certainly not optimal, in fact, in this case one expects $e_{1}-e_{0} \sim\left(\sigma m_{\omega}\right)^{-1}$.

3.4. The Poisson distribution of the obstacles. Let $z_{1}^{\omega} \leq z_{2}^{\omega} \leq \cdots \leq z_{m_{\omega}}^{\omega}$ denote those random points which lie in the unit interval $[0,1]$. The lengths $\ell_{i}=$ $z_{i+1}^{\omega}-z_{i}^{\omega}$ are independent random variables with distribution

$$
d P_{\nu}(\ell)=\nu e^{-\ell \nu} d \ell
$$

and we are considering the case $\nu \gg 1$. 
The average length of an interval free of obstacles is $\nu^{-1}$ and with probability one, $m_{\omega} / \nu \rightarrow 1$ and $\sum_{i} \ell_{i}=1$ for $\nu \gg 1$. Combined with the estimate on the energy gap this implies that the depletion of the BEC is uniform in the $L^{p}$ norm on sample space for any $p<\infty$.

3.5. Ideal vs interacting gas. While the average length of an interval is $\nu^{-1}$ there is, with probability one, a unique largest interval of length $\sim \nu^{-1} \ln \nu$. If there is no interaction, i.e., $\gamma=0$, and $\sigma \rightarrow \infty$ (Luttinger-Sy model), then the ground state wave function will be localized in the largest interval for kinetic energy reasons, with energy $\sim \nu^{2} /(\ln \nu)^{2}$.

The question is how the character of the wave function of the condensate, $\psi_{0}$, changes when the interaction, i.e., the term $(\gamma / N) \int\left|\psi_{0}(z)\right|^{4} d z$ comes into play.

\section{The Mass Distribution of the Condensate}

4.1. A limit theorem for the GP energy. Our first result is that the energy becomes deterministic in an appropriate limit.

Let $e_{\omega}(\gamma, \sigma, \nu)$ denote the GP energy with the random potential $V_{\omega}$ and $e_{0}(\gamma, \nu)$ the energy for $\sigma=\propto 2^{2}$, averaged over $\omega$.

THEOREM 2 (Convergence of the energy). Assume that $\nu \rightarrow \infty, \sigma \rightarrow \infty$ and $\gamma \rightarrow \infty$ in such a way that

$$
\gamma \gg \frac{\nu}{(\ln \nu)^{2}} \text { and } \sigma \gg \frac{\nu}{1+\ln \left(1+\nu^{2} / \gamma\right)} .
$$

Then, for almost every sample $\omega$,

$$
\lim \frac{e_{\omega}(\gamma, \sigma, \nu)}{e_{0}(\gamma, \nu)}=1
$$

4.2. Comments on the proof. The proof has two interrelated parts:

- Comparison of $e_{\omega}(\gamma, \sigma, \nu)$ with $e_{\omega}(\gamma, \infty, \nu)$.

- Comparison of $e_{\omega}(\gamma, \infty, \nu)$ with the deterministic $e_{0}(\gamma, \nu)$.

Calculations are conveniently done in a grand canonical ensemble, introducing a chemical potential $\mu$ that determines the optimal repartition

$$
n(\ell) \approx(\ell \gamma)^{-1}\left[\mu \ell^{2}-\pi^{2}\right]_{+}
$$

of the condensate mass in intervals of length $\ell$ between the obstacles. The lengths are distributed according to $d P_{\nu}(\ell)$ and the normalization requires

$$
\nu \int n(\ell) d P_{\nu}(\ell)=1 \text {. }
$$

4.3. Discussion of the GP wave function for large $\sigma$. For large $\sigma$ the average number of intervals with non-zero occupation numbers is given by

$$
\nu \int_{\pi / \sqrt{\mu}}^{\infty} d P_{\nu}(\ell)=e^{-\pi \nu / \sqrt{\mu}} \nu .
$$

Since $\nu$ is the total (average) number of available intervals,

$$
\lambda:=e^{-\pi \nu / \sqrt{\mu}} \leq 1
$$

\footnotetext{
${ }^{2}$ Recall that putting $\sigma=\infty$ amounts to requiring the wave functions to vanish at the positions of the obstacles.
} 
defines the fraction of them which are occupied. The normalization requires

$$
1 \sim \frac{\mu}{\gamma} e^{-\pi \nu / \sqrt{\mu}}
$$

so $\lambda$ is determined by $\gamma$ and $\nu$ via relation

$$
\frac{\lambda}{\left(\ln \lambda^{-1}\right)^{2}} \sim \frac{\gamma}{\nu^{2}}
$$

We can now distinguish the following limiting cases:

- If $\gamma \gg \nu^{2}$ then by (22) we get $\lambda \rightarrow 1$, i.e., all the intervals are occupied (delocalization). The chemical potential satisfies $\mu \sim \gamma$ in this regime.

- If $\gamma \sim \nu^{2}$ then $\lambda \sim 1$, but $\lambda$ is strictly less than 1 (transition to localization). Again we have $\mu \sim \gamma$.

- If $\gamma \ll \nu^{2}$ then $\lambda \ll 1$, i.e., only a small fraction of the intervals are occupied (localization). The relation (21) implies $\mu \sim \frac{\gamma}{\lambda}$ for the chemical potential.

- If $\gamma \sim \nu /(\ln \nu)^{2}$ then by (22) the fraction $\lambda$ becomes $O(1 / \nu)$, i.e., only finitely many intervals are occupied. In this latter case, $\mu \sim \gamma \nu \sim \nu^{2} /(\ln \nu)^{2}$, which corresponds exactly to the inverse of the square of the size of the largest interval.

In particular, $\lambda \nu \gg 1$ only if $\gamma \gg \nu /(\ln \nu)^{2}$, and hence this condition guarantees that many intervals are occupied. In this case the law of large numbers applies and hence the energy becomes deterministic in the limit.

If $\lambda \nu=O(1)$, on the other hand, the value of $e_{\omega}(\gamma, \sigma, \nu)$ is random. This shows, in particular, that our condition on $\gamma$ is optimal in the sense that for smaller $\gamma$ the energy fluctuates.

Also the condition $\sigma \gg \nu /\left(1+\ln \left(1+\nu^{2} / \gamma\right)\right)$ can be expected to be optimal. It can be rephrased as $\bar{\ell} \sigma \gg 1$, where $\bar{\ell}$ is the (weighted) average length of occupied intervals

$$
\bar{\ell}=\nu \int_{0}^{\infty} d P_{\nu}(\ell) \ell n(\ell)
$$

with $n(\ell)$ the optimal repartition of the mass. A simple calculation shows that $\bar{\ell} \sim \nu^{-1}\left(1+\ln \left(1+\nu^{2} / \gamma\right)\right)$.

4.4. Comments on scaling and the thermodynamic limit. Our model is formulated in the fixed interval $[0,1]$ so that the particle density $\rho$ tends to infinity as $N \rightarrow \infty$. Equivalently, we could have considered the model in an interval $[-L / 2, L / 2]$ and taking $N$ and $L \rightarrow \infty$ with $\rho=N / L$, as done for instance in [18. The two viewpoints are connected by simple scaling:

Let

$$
H_{L}=\sum_{i=1}^{N}\left(-\partial_{i}^{2}+b \sum_{j} \delta\left(x_{i}-x_{j}^{\omega}\right)\right)+g \sum_{i<j} \delta\left(x_{i}-x_{j}\right)
$$

be the Hamiltonian on the Hilbert space $L^{2}([-L / 2, L / 2], d x)^{\otimes_{s} N}$ with $x_{j}^{\omega}$ Poisson distributed with density $d$. Writing $x_{i}=L z_{i}-(L / 2)$ transforms $H_{L}$ into $L^{-2} H$ with $H$ the Hamiltonian (11) on $[0,1]$ and

$$
\sigma=L b, \quad \nu=L d, \quad \gamma=L N g=L^{2} \rho g .
$$

The condition for BEC in Theorem 3.1 as well as the conditions (15) for the convergence of the GP energy to a deterministic value can straightforwardly be written 
as conditions for the parameters $b, d$ and $\rho g$ as $L \rightarrow \infty$. In particular, if $b$ and $d$ are fixed, then (15) is fulfilled provided

$$
\left(L(\ln L)^{2}\right)^{-1} d \ll \rho g \ll d^{2} .
$$

Note also that the validity of the GP approximation requires in any case that the dimensionless parameter $g / \rho$ is $\ll 1$, cf., e.g., [26].

\section{Conclusions}

- BEC in the ground state of the interacting gas in the GP regime can survive even in a strong random potential. The character of the wave function of the condensate, however, is strongly affected by the interaction.

- A random potential may lead to localization of the wave function of the condensate in subintervals. The interparticle interaction counteracts this effect, however, and can lead to complete delocalization (the condensate extends over the whole unit interval) if the interaction is strong enough.

- In terms of the interaction strength, $\gamma$, and density of scatterers, $\nu$, the transition between localization and delocalization occurs in the model considered when $\gamma \sim \nu^{2}$. For $\gamma \lesssim \nu /(\ln \nu)^{2}$ a "third phase" occurs where the condensate is localized in a small number of subintervals.

\section{REFERENCES}

[1] L.A. Pastur, A. Figotin, Spectra of Random and Almost-Periodic Operators, Springer-Verlag, 1992

[2] O. Lenoble, L.A. Pastur, V.A. Zagrebnov, Bose-Einstein condensation in random potentials, C.R. Physique 5, 129-142 (2004)

[3] O.Lenoble, V.A.Zagrebnov, Bose-Einstein Condensation in the Luttinger-Sy Model, Markov Proc.Rel.Fields 13, 441-468 (2007).

[4] Th.Jaeck, J.V.Pulé, V.A.Zagrebnov, On the Nature of Bose-Einstein Condensation Enhanced by Localization, J.Math.Phys. 51, 103302-15 (2010).

[5] H. Gimperlein, S. Wessel, J. Schmiedmayer, L. Santos, Ultracold Atoms in Optical lattices with Random One-Site Interactions, Phys. Rev. Lett. 95, 170401 (2005)

[6] V.I. Yukalov, R. Graham, Bose-Einstein condensed systems in random potentials, Phys. Rev. A 75, 023619 (2007)

[7] P. Lugan, P. Bouyer, A. Aspect, M. Lewenstein, L. Sanches-Palencia, Ultracold Bose Gases in $1 D$ Disorder: From Lifshits Glass to Bose-Einstein Condensate, Phys. Rev. Lett. 98, 170403 (2007)

[8] L. Fallani, C. Fort, M. Inguscio, Bose-Einstein Condensates in Disordered Potentials, Adv. At. Molec. Opt. Phys. 56, 119-159 (2008)

[9] L. Sanches-Palencia, D. Clément, P. Lugan, P. Bouyer and A. Aspect, Disorder-induced trapping versus Anderson localization in Bose-Einstein condensates expanding in disordered potentials, New J. Phys. 10, 045019 (2008)

[10] A.S. Pikovsky, D.L. Shepelyansky, Destruction of Anderson Localization by a Weak Nonlinearity, Phys. Rev. Lett. 100, 094101 (2008)

[11] P. Lugan, A. Aspect, L. Sanches-Palencia, D. Delande, B. Grémaud, C.A. Müller, C. Miniatura, One-dimensional Anderson localization in certain correlated random potentials, Phys. Rev. A 80, 023605 (2009)

[12] J. Radic, V. Bacic, D. Judic, M. Segev, H. Buljan, Anderson localization of a Tonks-Girardeau gas in potentials with controlled disorder, Phys. Rev. A 81, 063639 (2010)

[13] I.L. Aleiner, B.L. Altshuler, G.V. Shlapnykov, A finite-temperature phase transition for disordered weakly interacting bosons in one dimension, Nature Phys. 6, 900-904 (2010)

[14] L. Sanches-Palencia, M. Lewenstein, Disordered quantum gases under control, Nature Phys., 6, 87-95 (2010)

[15] M. Piraud, P. Lugan, B. Bouyer, A. Aspect, and L. Sanches-Palencia, Localization of a matter wave packet in a disordered potential, Phys. Rev. A 83, 031603(R) (2011) 
[16] W.B. Cardoso, A.T. Avelar, D. Bazeia, Anderson localization of matter waves in chaotic potentials, Nonlin. Analysis 13, 755-763 (2012)

[17] J. Stasinska, P. Massingnan, M. Bishop, J. Wehr, A. Sanpera and M. Lewenstein, The glass to superfluid transition in dirty bosons on a lattice, New J. Phys. 14, 043043 (2012)

[18] M. Bishop, J. Wehr, Ground State Energy of Mean-field Model of Interacting Bosons in Bernoulli Potential, arXiv:1212.1487

[19] R. Seiringer, J. Yngvason and V. A. Zagrebnov, Disordered Bose-Einstein condensates with interaction in one dimension, J. Stat. Mech. P11007 (2012), arXiv:1207.7054

[20] E.H. Lieb, W. Liniger, Exact Analysis of an Interacting Bose Gas. I., Phys. Rev. 130, 1605$1616(1963)$

[21] J. Blank, P. Exner, M. Havlíček, Hilbert Space Operators in Quantum Physics, SpringerVerlag, 2008

[22] J.M.Luttinger, H.K.Sy, Bose-Einstein Condensation in a One-Dimensional Model with Random Impurities, Phys. Rev. A 7, 712-720 (1973)

[23] E.H. Lieb, R. Seiringer, Proof of Bose-Einstein Condensation for Dilute Trapped Gases, Phys. Rev. Lett. 88, 170409 (2002)

[24] B. Baumgartner, J.-P. Solovej and J. Yngvason, Atoms in Strong Magnetic Fields: the High Field Limit at Fixed Nuclear Charge, Commun. Math. Phys. 212, 703-724 (1998)

[25] W. Kirsch, B. Simon, Universal lower bounds on eigenvalue splittings for one dimensional Schrödinger operators, Commun. Math. Phys. 97, 453-460 (1985)

[26] E.H. Lieb, R. Seiringer and J. Yngvason, One Dimensional Behavior of Dilute, Trapped Bose Gases, Commun.Math.Phys. 244, 347-393 (2004)

Department of Mathematics and Statistics, McGill University, 805 Sherbrooke Street West, Montreal, QC H3A 2K6, Canada, E-mail: robert.Seiringer@MCGill.CA

Fakultät für Physik, Universität Wien,, Boltzmanngasse 5, 1090 Vienna, Austria, E-MAIL: JAKOB.YNGVASON@UNIVIE.AC.AT

Département de Mathématiques, Université d'Aix-Marseille (AMU) and Centre de Physique Théorique - UMR 7332, Luminy Case 907, 13288 Marseille, Cedex09, France, E-MAIL: VALENTIN.ZAGREBNOV@UNIV-AMU.FR 